\title{
COMPLETELY 0-SIMPLE AND HOMOGENEOUS $n$ REGULAR SEMIGROUPS
}

\author{
JIN BAI KIM
}

1. The purpose of this paper is to generalize R. McFadden and Hans Schneider's Theorem [3].

2. Definition and notation. Let $a \neq 0$ be a regular element of a semigroup $S$. An element $x$ in $S$ is called an inverse of $a$ if $a x a=a$ and $x a x=x$. Let $n$ be a fixed positive integer. A semigroup $S$ with zero is said to be homogeneous $n$ regular if every nonzero element of $S$ has precisely $n$ distinct inverse elements in $S$. A semigroup $S$ with zero is said to be null if $S S=\{0\}$. A semigroup $S$ will be called a right (left) zero semigroup if $x y=y(x y=x)$ for all $x, y$ in $S .|T|$ denotes the cardinality of a set $T$.

If $S$ is completely 0 -simple, I shall follow Clifford-Preston [1] (with $J$ replacing $\Lambda$ ) and let $\left\{R_{i}: i \in I\right\}$ be the set of nonzero $R$ classes, $\left\{L_{j}: j \in J\right\}$ the set of nonzero $L$-classes, $\left\{H_{i j}=R_{i} \cap L_{j}:(i, j)\right.$ $\in(I x J)\}$, be the set of nonzero $H$-classes and write $R_{i}^{0}=R_{i} \cup\{0\}$. If $a \neq 0$ is in a semigroup $S, E_{a}=\left\{e \in S: e=e^{2}\right.$ and $\left.e a=a\right\}, F_{a}=$ $\left\{f \in S: f=f^{2}\right.$ and $\left.a f=a\right\}, N_{a}=\{x \in S: a x a=a$ and $x a x=x\}, h(i)$ $=\mid\left\{j \in J: H_{i j}\right.$ is a group $\} \mid$ and $k(j)=\mid\left\{i \in I: H_{i j}\right.$ is a group $\} \mid$. If $T \subseteq S, \varepsilon(T)=\left\{e \in T: e=e^{2}\right\}$. A homogeneous $n$ regular semigroup $S$ is called an $(h, k)$ type if for all $a \in S \backslash 0,\left|E_{a}\right|=h$ and $\left|F_{a}\right|=k$, where $h$ and $k$ are fixed positive integers with $h k=n$.

3. We shall need the following lemmas.

Lemma A. Let $S$ be completely 0-simple. If $a \in H_{i j}$, then

(1) $E_{a}=\mathcal{E}\left(R_{i}\right)$ and $\left|E_{a}\right|=h(i)$.

(2) $F_{a}=\varepsilon\left(L_{j}\right)$ and $\left|F_{a}\right|=k(j)$.

(3) $\left|N_{a}\right|=h(i) k(j)$.

Proof. (1) Since $H_{i k}, k \in J$ contains an idempotent if and only if $H_{i k}$ is a group, $\left|\mathcal{E}\left(R_{i}\right)\right|=h(i)$. By Lemma 2.14 of $[1], \varepsilon\left(R_{i}\right) \subseteq E_{a}$. If $e \in E_{a}$, then obviously $\{0\} \neq e S \subseteq a S \subseteq R_{i}^{0}$ whence $E_{a} \subseteq \mathcal{E}\left(R_{i}\right)$. Hence $E_{a}=\varepsilon\left(R_{i}\right)$ and (1) follows. The proof of (2) is similar.

As an immediate application of [1, Theorem 2.18], we see that $a \in H_{i j}$ has an inverse in $H_{m n}$ if and only if both $H_{m j}$ and $H_{i n}$ are groups, and in this case the inverse in $H_{m n}$ is unique. Thus (3) follows.

Received by the editors May 6, 1965. 
Lemma B. For all $a, b$ in a completely 0-simple semigroup $S$ aba $=a \neq 0$ implies $b a b=b$.

Proof. Let $a x a=a \neq 0$. Then $\{0\} \neq S(a x)=S x$ whence $a x$ is a right identity of $S x$ and $x \in S x$. Hence $x a x=x$.

4. The following theorem is a generalization of R. McFadden and Hans Schneider's Theorem.

Theorem. Let $S$ be a 0 -simple semigroup and let $n$ be a positive integer. Then there exist positive integers $h$ and $k$ such that $n=h k$ and for which the following statements are equivalent.

(i) $S$ is an $(h, k)$ type homogeneous $n$ regular and completely 0 -simple semigroup.

(ii) For every $a \neq 0$ in $S$ there exist precisely $n$ distinct nonzero elements $\left\{x_{i}\right\}_{i=1}^{n}$, such that $a x_{i} a=a$ for $i=1,2, \cdots, n$, and for $c, d$ in $S c d c=c \neq 0$ implies $d c d=d$.

(iii) For every $a \neq 0$ in $S$ there exist precisely $h$ distinct nonzero idempotents $\left\{e_{i}\right\}_{i=1}^{h}=E_{a}$ and $k$ distinct nonzero idempotents $\left\{f_{j}\right\}_{i=1}^{\boldsymbol{k}}=F_{a}$ such that $E_{a} \cap F_{a}$ contains at most one element.

(iv) Every nonzero principal right ideal $R$ contains precisely $h$ nonzero idempotents and every nonzero principal left ideal $L$ contains precisely $k$ nonzero idempotents such that $R \cap L$ contains at most one nonzero idempotent.

(v) $S$ is completely 0 -simple. For every 0 -minimal right ideal $R$ there exist precisely $h$ 0-minimal left ideals $\left\{L_{i}\right\}_{i=1}^{h}$ and for every 0 minimal left ideal $L$ there exist precisely $k$ 0-minimal right ideals $\left\{R_{j}\right\}_{j=1}^{\boldsymbol{k}}$ such that $L R_{j}=L_{i} R=S$, for every $i=1,2, \cdots, h, j=1$, $2, \cdots, k$.

(vi) $S$ is completely 0-simple. Every 0-minimal right ideal $R$ is the union of a right group with zero $G^{0}$, a union of h disjoint groups except zero, and a null subsemigroup $Z$ which annihilates the right ideal $R$ on the left and every 0-minimal left ideal $L$ is the union of a left group with zero $G^{\prime \prime}$, a union of $k$ disjoint groups except zero, and a null subsemigroup $Z^{\prime}$ which annihilates the left ideal $L$ on the right.

(vii) $S$ contains at least $n$ nonzero distinct idempotents, and for every nonzero idempotent $e$ there exists a set $E=\left\{e_{i}\right\}_{i=1}^{n}$ of nonzero idempotents of $S$ such that $e E$ is a right zero subsemigroup of $S$ containing precisely h nonzero idempotents, Ee is a left zero subsemigroup of $S$ containing precisely $k$ nonzero idempotents, $e(\mathcal{E}(S) \backslash E)=\{0\}=(\mathcal{E}(S) \backslash E) e$, and $e E \cap E e=\{e\}$.

REMARK 1. If $n=1$, then the theorem above takes the same form as R. McFadden and Hans Schneider's Theorem [3]. 
5. Proof of the theorem. (i) implies (ii). This is clear by the definition of an $(h, k)$ type homogeneous $n$ regular semigroup and Lemma B. (ii) implies (iii). We shall prove the existence of a nonzero primitive idempotent of $S$. Let $a$ be a nonzero element of $S$. By (ii) there exist $\left\{x_{i}\right\}_{i=1}^{n}$ in $S$ such that $a x_{i} a=a$ and $x_{i} a x_{i}=x_{i}$ for every $i=1,2, \cdots, n$.

Choose $a x_{1}=e$. Clearly $0 \neq e \in \mathcal{E}(S)$. Let $f$ be any nonzero idempotent such that $f e=e f=f$. Since $f e f=(f e) f=f f=f$, we have $e f e=e$ by the assumption of (ii). But we have $e f e=e(f e)=e f=f$. Hence we conclude $e=f$, and $e$ is a nonzero primitive idempotent of $S$ and hence $S$ is completely 0 -simple [1, p. 76]. The last assertion of (iii) now follows since each $H$-class has at most one idempotent.

Let $a \in H_{i j}$ and $b \in H_{m q}$. Define $h=h(m)$ and $k=k(q)$. Let $c \in H_{i q}$ and $d \in H_{m i}$. By Lemma A and (ii)

$$
\begin{aligned}
& n=\left|N_{a}\right|=h(i) k(j), \\
& n=\left|N_{c}\right|=h(i) k(q)=h(i) k, \\
& n=\left|N_{b}\right|=h(m) k(q)=h k .
\end{aligned}
$$

Thus it follows that $h=h(i), k=k(j),\left|E_{a}\right|=h$, and $\left|F_{a}\right|=k$. (iii) implies (iv). By (iii), $S$ contains nonzero idempotent. Let $e, f$ be nonzero idempotents such that $e f=f e=f$. Then both $e, f$ are in $E_{f} \cap F_{f}$, whence $e=f$. Hence $S$ is completely 0 -simple. The rest is just Lemma A, parts (1), (2).

(iv) implies (v). By (iv), it is clear that $S$ has a nonzero primitive idempotent, and hence $S$ is completely 0 -simple. Then every nonzero principal right ideal $R(a)=a \cup a S=a S$ for $a \neq 0$ in $S$ is a 0 -minimal right ideal of $S$ by Exercise 2 in [1, p. 83]. Let $\&(R(a) \backslash 0)=\left\{e_{i}\right\}_{i=1}^{h}$ and let $L_{i}=S e_{i}$. Then $\left\{L_{i}\right\}_{i=1}^{h}$ are 0 -minimal left ideals of $S$ such that $L_{i} R(a)=S(i=1,2, \cdots, h)$ by $[1$, Lemma 2.46$]$. The proof for a 0 -minimal left ideal $L(a)=S a$ is analogous. (v) implies (vi). Let $R$ be a 0 -minimal right ideal of $S$. Then by $(\mathrm{v})$ there exists a set $\left\{L_{i}\right\}_{i=1}^{h}$ of 0 -minimal left ideals such that $L_{i} R=S(i=1,2, \cdots, n)$. By [1, Lemma 2.46], $R \cap L_{i}=R L_{i}$ is a group with zero. Let $G^{0}=\bigcup_{i=1}^{h}\left(R L_{i}\right)$ and let $Z$ be the complement of the nonzero part of $G^{0}$ in $R$. Then $R=G^{0} \cup Z$, and $Z R=\{0\}$ since each element of $Z$ belongs to a 0 -minimal left ideal $L^{\prime}$ for which $L^{\prime} R=\{0\}$ by [1, Lemma 2.46]. Therefore $Z$ is a null subsemigroup of $S$. By [1, Exercise 2, p. 39], it suffices to show that $\mathcal{E}(G)=\varepsilon\left(G^{0} \backslash 0\right)$ is a right zero semigroup. From [1, Lemma $2.43]$, it follows that $\varepsilon(R \backslash 0)$ is a right zero semigroup, and so is $\varepsilon(G)$.

The proof of the rest is similar to the proceeding argument.

(vi) implies (vii). Assume (vi). Let $e \in \mathcal{E}(S \backslash 0)$ and let $\mathcal{E}(e S \backslash 0)$ 
$=\left\{e_{i}\right\}_{i=1}^{h}$. Define $E=\bigcup_{i=1}^{h}\left(S e_{i} \backslash 0\right)$. Then $|E|=h k=n$. From $E e \subset S e$ and $E e \subseteq \varepsilon(S e)$ it follows that $E e$ is a left zero semigroup with $|E e|=k$ $=|\mathcal{E}(S e \backslash 0)|$. We claim that $(\varepsilon(S) \backslash E) \cdot e=\{0\}$.

Assume, by way of contradiction, that $g e \neq 0$ for some $g$ in $(\varepsilon(S) \backslash E)$. Setting $L=S g$ and $R=e S$, we have that $R L=R \cap L$ is a group with zero by Lemma 2.46, [1]. Then $g \in \mathcal{E}(L) \subseteq E$, contrary to $g \in \mathcal{E}(S) \backslash E$. Thus we must have $(\mathcal{E}(S) \backslash E) \cdot e=\{0\}$. Analogously, we can show that $e \cdot E$ is a right zero semigroup, $|e E|=h$ and $e \cdot(\varepsilon(S) \backslash E)=\{0\}$. Finally, from $(e E \cap E e) \subset(e S \cap S e)=H_{e}^{0}$, it follows that $e E \cap E e=\{e\}$. (vii) implies (i). If $e f=f e=f 0 \neq f=f^{2}$ then $f \in E$ by $e(\varepsilon(S) \backslash E)=\{0\}$, whence $f \in e E \cap E e=\{e\}$. Thus $e=f$, and $S$ is completely 0 -simple. Suppose $e S \backslash 0=R_{i}$. Since $e(\varepsilon(S) \backslash E)=\{0\}$, it follows that $\varepsilon\left(R_{i}\right) \subseteq E$, whence $\mathcal{E}\left(R_{i}\right) \subseteq e E$. But as $e E$ is a right zero subsemigroup each $g \in e E$ is idempotent. Also $0 € e E$, for since $e \in e E, x e=e$, all $x \in e E$. Hence $e E \subseteq \mathcal{E}\left(R_{i}\right)$. We have proved that $\mathcal{E}\left(R_{i}\right)=e E$. Let $0 \neq a \in H_{i j}$. There exists an idempotent $e \in R_{i}$. Then $e E=\varepsilon\left(R_{i}\right)=E_{a}$, by Lemma $\mathrm{A}$, whence $\left|E_{a}\right|=|e E|=h$ by (vii). Similarly, $\left|F_{a}\right|=k$. By Lemma A, $\left|N_{a}\right|=h \cdot k=n$ and (i) is proved.

This completes the proof of the theorem.

REMARK 2. In the theorem above, $h, k$, and $n$ could be infinite cardinals.

6. I wish to thank a referee for his suggestions. The results in this paper form a portion of the author's doctoral dissertation written at the Virginia Polytechnic Institute under the direction of Professors P. H. Doyle and Rebecca Slover, to whom the author wishes to express his appreciation.

\section{REFERENCES}

1. A. H. Clifford and G. B. Preston, The algebraic theory of semigroups, Vol. I, Math. Surveys No. 7, Amer. Math. Soc., Providence, R. I., 1961. 526.

2. —_ Semigroups containing minimal ideals, Amer. J. Math. 70 (1948), 521-

3. R. McFadden and Hans Schneider, Completely simple and inverse semigroups, Proc. Cambridge Philos. Soc. 57 (1961), 234-236.

4. W. D. Munn, Brandt congruences of inverse semigroups, Proc. London Math. Soc. 14 (1964), 154-164.

5. P. S. Venkatesan, On a class of inverse semigroups, Amer. J. Math. 84 (1962), 578-582.

Virginia Polytechnic Institute and Michigan State University 